\title{
Invited commentary on ... When unbearable suffering incites psychiatric patients to request euthanasia ${ }^{\dagger}$
}

\author{
Brendan D. Kelly
}

\section{Summary}

Euthanasia is available in Belgium and Luxembourg for untreatable and unbearable suffering resulting from 'physical and/or psychological suffering that cannot be alleviated and results from a serious and incurable disease, caused by accident or illness'. Verhofstadt et al's valuable analysis of testimonials from psychiatric patients requesting euthanasia demonstrates that elements of this suffering might well be alleviated. We should not kill our patients.

\section{Declaration of interest}

None.

\section{Copyright and usage}

(c) The Royal College of Psychiatrists 2017.
The issues raised by euthanasia for mental illness and physicianassisted suicide for psychiatric patients are complicated, controversial and important. The legal situations vary greatly between countries and the interesting paper by Verhofstadt et al emanates from one of the few countries that permits euthanasia for mental illness: Belgium. ${ }^{1}$ As the authors point out, euthanasia and/or physician-assisted suicide is legal in just five of the United States, four European countries (The Netherlands, Belgium, Luxembourg and Switzerland) and Colombia. The authors write that criteria in Belgium and Luxembourg include untreatable and unbearable suffering, stemming from 'physical and/or psychological suffering that cannot be alleviated and results from a serious and incurable disease, caused by accident or illness'. This includes mental illness.

\section{Should euthanasia ever be countenanced for mental illness alone?}

Most readers of the paper by Verhofstadt et al will not reside in a country in which euthanasia is provided for mental illness alone. As a result, this careful, interesting paper will surprise many readers and offer new, possibly disturbing insights to some. In this context, and before proceeding to the specific findings presented by Verhofstadt et al, the key, over-arching issue merits consideration: should euthanasia or physician-assisted suicide ever be provided for suffering resulting from mental illness alone?

Even if physician-assisted suicide is permitted for extreme suffering resulting from a physical disorder (such as metastatic cancer), should it also be permitted for mental illness that can include suicidality as an intrinsic feature of the illness itself? If an otherwise physically healthy 16-year old develops depression and decides she or he wants to die, are there any circumstances in which a psychiatrist should ever countenance actively assisting with such a request?

There are many problems with such a course of action. In the first instance, it is exceptionally difficult to state - as required by the Belgian criteria - that suffering resulting from mental illness 'cannot be alleviated' or that the disorder is 'incurable'. Although treatment-resistant cases certainly do occur in psychiatry, it is also

${ }^{\dagger}$ See pp. 238-245 and 246-247, this issue. true that treatments evolve, circumstances shift, and people change, often resulting in unexpected improvements over time. The only exceptions in psychiatry might be in certain cases of advanced dementia (such as advanced Alzheimer's) in which cases the biological basis of the disorder is far better understood than, for example, treatment-resistant depression, making prognostication more reliable. ${ }^{2}$

Even so, clinical courses in most mental illnesses are far more unpredictable than those in many physical disorders, such as terminal, metastatic cancer. The fact that the biological basis of mental illnesses is generally poorly understood also marks them out as qualitatively different to physical disorders. The apparent psychological determinants of certain mental illnesses may well be more amenable to change than many of the known determinants of established cancer.

There is also a profound medical ethical issue here, and it is simply insufficient for a medical practitioner to seek refuge in the fact that the laws of a given country permit euthanasia for extreme suffering resulting from mental illness. Doctors' actions need to accord not only with the law of the land but also with sound principles of medical ethics that provide additional guidance and protections to both patients and doctors in complex situations.

Virtually all of medical practice and mental healthcare is geared towards the preservation and/or enhancement of life. This principle is written into the medical ethical codes of many countries. For example, the Guide to Professional Conduct and Ethics for Registered Medical Practitioners in Ireland, specifies that 'you must not take part in the deliberate killing of a patient. ${ }^{3}$ In the UK, the General Medical Council outlines a 'presumption in favour of prolonging life':

'Following established ethical and legal (including human rights) principles, decisions concerning potentially life-prolonging treatment must not be motivated by a desire to bring about the patient's death, and must start from a presumption in favour of prolonging life. This presumption will normally require you to take all reasonable steps to prolong a patient's life. However, there is no absolute obligation to prolong life irrespective of the consequences for the patient, and irrespective of the patient's views, if they are known or can be found out'. ${ }^{4}$

\section{Verhofstadt et al's contribution to the debate}

The situation is different in Belgium and it is from this context that the paper by Verhofstadt et al has emerged. For the purpose 
of the paper, the authors do not explore the fundamental wrongness or rightness of the Belgian legislation in depth, but move on to study the concept of 'unbearable suffering' based on testimonials from 26 psychiatric patients with a death request. The various methodological strengths and weaknesses of the study are explored in the paper, which merits close reading.

For many readers, one of the most striking features of the paper will be the impression that at least some of the causes of suffering that reportedly 'cannot be alleviated' appear, in fact, to be amenable to alleviation. The section on 'suffering related to one's place and interaction in society', for example, cites 'low income' as contributing to the 'unbearable suffering' that ultimately led to the request for euthanasia. In one way, this makes perfect sense: human suffering is invariably multifactorial, and the suffering resulting from illness is admixed with other causes of suffering, such as poverty. But surely 'low income' is sufficiently fixable that it should never contribute (directly or indirectly) to a request for euthanasia? Or if it does, surely the most compassionate response to that element of suffering is to provide social or economic assistance, rather than assisting with death? And it is especially disturbing to read that one of the identified problems with 'low income' was that it necessitated 'careful consideration to determine whether alternative admissions and treatments (i.e. non-psychiatric) were feasible'. Does this mean some patients requested euthanasia in part because it was the only intervention they could afford? If so, that is horrifying. The authors duly highlight that 'a wider political and societal debate may be needed to find ways to reduce the medical costs and/or improve the financial situation of certain individuals in order to reduce the desire to request euthanasia'.

It is also disturbing to read that 'feelings of hopelessness and [ . . . ] incurability appeared as patients felt or were told by their physicians that their suffering could not be alleviated or that there was little - if any - hope for recovery or even improvement'. In the vast majority of cases of mental illness, prognosis is notoriously difficult to predict and it is impossible to say for certain that there is no hope for improvement.

That is, perhaps, the key issue that this paper identifies: that at least some of the suffering that reportedly 'cannot be alleviated' can, in fact, be alleviated. There is always hope. Human suffering is complex, multifactorial and continually evolving. With mental illness, there is no point at which we can say that a person's illness is untreatable or that their suffering cannot be alleviated, either now or at some future point.

\section{Conclusions}

Euthanasia or physician-assisted suicide should not be provided for mental illness alone. We should acknowledge extreme distress when it occurs and remain focused on providing compassionate, life-affirming care.

We should not kill our patients.

Brendan D. Kelly, MB, BCh, BAO, MA, MSC, MA, MA, MD, PhD DGov, PhD CPsychol, FRCPI, FRCPsych, FTCD, Department of Psychiatry, Trinity College Dublin, Trinity Centre for Health Sciences, Tallaght Hospital, Dublin D24 NROA, Ireland. Email: brendan.kelly@tcd.ie

First received 5 Mar 2017, final revision 25 Jun 2017, accepted 20 Jul 2017

\section{Acknowledgements}

This paper contains public sector information licensed under a Creative commons Attribution 4.0 International (CC BY 4.0) licence (Ireland) and the Open Government Licence v.3.0 (United Kingdom).

\section{References}

1 Verhofstadt $M$, Thienpont L, Peters G-JY. When unbearable suffering incites psychiatric patients to request euthanasia: qualitative study. Br J Psychiatry 2017; 211: 238-45.

2 Kelly BD, McLoughlin DM. Euthanasia, assisted suicide and psychiatry: a Pandora's box. Br J Psychiatry 2002; 181: 278-9.

3 Medical Council. Guide to Professional Conduct and Ethics for Registered Medical Practitioners (8th edn). Medical Council (Ireland), 2016.

4 General Medical Council. Treatment and Care Towards the End of Life: Good Practice in Decision Making. General Medical Council, 2010. 